\title{
La formación en investigación educativa de los futuros profesores
}

\author{
Haylen A. Perines \\ Instituto de Investigación Multidisciplinario de Ciencia y Tecnología, Universidad de La Serena, Avenida Raúl Bitrán \\ Nachary s/n La Serena, Chile (correo-e: haylen.perines@userena.cl).
}

Recibido Dic. 12, 2019; Aceptado Feb. 5, 2020; Versión final Mar. 20, 2020, Publicado Ago. 2020

\begin{abstract}
Resumen
La presente investigación tiene como objetivo analizar las valoraciones que los docentes universitarios de las pedagogías tienen sobre la formación en investigación educativa de los futuros profesores. Se presentan también los primeros resultados del proyecto FONDECYT: "presencia e importancia de la investigación educativa en la formación pedagógica y su valoración por estudiantes y docentes". Se realizó un enfoque mixto con docentes de la Universidad de La Serena (Chile), quienes respondieron un cuestionario y una entrevista semi-estructurada. Los resultados muestran que los docentes universitarios consideran la formación en investigación educativa como un elemento fundamental en la preparación profesional de futuros profesores. Se identifican desafíos pendientes como optimizar su propia actitud hacia la investigación educativa para instaurar una cultura investigativa en las universidades. Se concluye que es importante que los académicos optimicen su propia actitud hacia la investigación educativa y que se intente instaurar una cultura investigativa al interior de las universidades.
\end{abstract}

Palabras clave: formación docente; universidad; investigación educativa; aprendizaje de la investigación

\section{Training in educational research for future professors}

\begin{abstract}
The main objective of this research study is to analyze the assessment of university pedagogy professors about the training on educational research of future professors. The present study also presents the first results from the FONDECYT project: "presence and importance of educational research in pedagogical training and its assessment by students and teachers." A mixed-focus study is performed with professors from the University of La Serena (Chile), who answered a questionnaire and participated in a semi-structured interview. The results suggest that university professors consider training on educational research as a fundamental element on the professional development of future professors. However, the results show pending challenges such as optimizing the professors' attitude towards educational research. In conclusion, this research study highlights the importance for professors to improve their own attitudes towards educational research in order to establish a research culture within universities.
\end{abstract}




\section{INTRODUCCIÓN}

Es comúnmente aceptado que la enseñanza basada en la investigación puede mejorar la calidad de la educación y, posteriormente, la práctica de los profesionales (Heggen et al., 2010). Esto ha posibilitado que exista un fuerte movimiento en la educación superior para aumentar la experiencia de investigación de los estudiantes y contribuir así a una formación investigativa (Spronken-Smith et al., 2012, Cardoso y Cerecedo, 2019; Lazcano-Peña, Viedma y Alcaino, 2019). La formación de profesorado no ha estado ausente de este nuevo contexto, que se complementa con la llamada crisis de la investigación educativa (Kaestle, 1993). Dicho periodo de cuestionamiento, que incluyó la comparación crítica entre la medicina y la enseñanza (Hargreaves, 1996) y las dudas sobre el impacto de la investigación educativa en la práctica docente (Díaz et al., 2019) posibilitó el surgimiento de la línea de investigación que estudia las valoraciones que el profesorado -tanto en formación como en ejercicio- tiene sobre la investigación educativa (Bartels, 2003; Demircioglu, 2008; Haberfellner y Fenzl, 2017; Perines y Murillo, 2017). Sin embargo, existen pocas evidencias sobre las valoraciones que los docentes universitarios tienen sobre la formación en investigación educativa, más aún en el contexto de preparación profesional de los futuros profesores. En este escenario, el objetivo general del presente artículo es analizar las valoraciones que los profesores universitarios de las pedagogías tienen sobre la formación en investigación educativa de los futuros docentes.

Analizar el rol de la formación en investigación educativa es urgente, considerando, por ejemplo, las críticas que los profesores en ejercicio hacen sobre su formación inicial en materias de investigación (p.ej., Aldana de Becerra, 2012; Murillo, Perines y Lomba, 2017). Al respecto, Anwaruddin y Pervin (2015) señalan que los docentes en ejercicio no se comprometen con la investigación porque no han sido "alfabetizados" cuando estaban en la formación inicial. En esta línea, cobra sentido lo que afirman Rubio, et al. (2018) "formar profesionales que utilicen los métodos de la ciencia para transformar la realidad es una necesidad". La presente revisión teórica se aborda desde dos perspectivas. La primera de ellas se enfoca en los estudios existentes sobre valoraciones de profesores universitarios en torno a la investigación, a la formación en investigación educativa y al rol del profesor-investigador (Broekkamp y van Hout-Wolters, 2007; Joram, 2007; Larenas et al., 2015; Schouteden et al., 2016). La segunda perspectiva se refiere a las investigaciones que, a la fecha, han dado cuenta de la importancia de dicha formación en el profesorado (Admiraal et al., 2017; Barkhuizen et al., 2018; Flores et al., 2016).

Respecto al primer tema, el estudio de Schouteden et al. (2016), realizado con 79 profesores de instituciones de educación superior, concluyen que los participantes emiten opiniones más bien rígidas y tradicionales sobre la investigación, para después pasar a interpretaciones matizadas por elementos de su práctica. En este punto es interesante preguntarse cuál es el rol que los investigadores juegan en generar esta integración práctica. Al respecto, Broekkamp, H. y van Hout-Wolters, (2007) concluyen que se requiere de una mejor colaboración con los investigadores para acercar la investigación a la práctica. En dicha investigación se establece una diferencia entre ser profesor universitario y ser investigador, idea que va en coherencia a lo postulado por autores como Roche y Marsh (2000) y Marsh y Hatie (2002). Sin embargo, esta mirada ha sido refutada por otros autores. Por ejemplo, para Hernández Arteaga (2011) "la universidad debe promover la apertura de espacios académicos, donde la investigación sea la base de la docencia” (p. 4). Al respecto, y en un estudio con 259 profesores universitarios, Tesouro et al. (2014), concluyen que la identidad profesional y el equilibrio entre las diferentes funciones del profesor universitario están influidos por el contexto en el cual este actúa y que solo se conseguirá el nexo auténtico si los profesores desean que su trabajo docente e investigador esté relacionado. Asimismo, en el estudio de Larenas et al. (2015), que incluye a profesores del sistema escolar y profesores universitarios, un $65 \%$ de los docentes universitarios plantea que enseña herramientas investigativas a sus estudiantes, pero solo un $28 \%$ señala que está formando a futuros investigadores.

Por otra parte, en las investigaciones sobre la importancia de una formación en investigación educativa del profesorado existen estudios que analizan esta temática desde la perspectiva de los profesores en ejercicio y otros que lo hacen desde la visión de la formación inicial docente. Desde la perspectiva de los profesores en ejercicio, Admiraal et al. (2017) realizan un estudio en el cual cuatro profesores de secundaria investigaron sus clases, guiados por un profesor universitario. Posteriormente, los docentes respondieron algunas interrogantes que descifraron la presencia de tres temas fundamentales: gracias al trabajo de investigación habían realizado cambios significativos en la forma en que enseñan y piensan acerca de la enseñanza, reemplazaron o complementaron algunos textos populares por artículos de revistas científicas y ampliaron de la base de conocimientos sobre enseñanza y aprendizaje.

Ideas similares mencionan Barkhuizen et al. (2018) cuando publican un libro centrado en cómo los profesores pueden convertirse en investigadores de sus propias aulas. De acuerdo con los autores, los profesores que hacen investigación tienen una mayor apreciación de la relación entre la teoría y la práctica y una reflexión más crítica sobre el impacto de sus prácticas en el aula. En esta línea, Burns et al. (2017) y Smith (2018) 
instauran el lema "investigación por docentes para docentes y sus alumnos", que han tenido lugar en diferentes contextos nacionales en Turkía. En un estudio reciente, van Schaik et al. (2018) analizaron 66 artículos de investigación para identificar las barreras que los docentes tienen para utilizar el conocimiento académico y qué condiciones mejoran dicha utilización. Entre los resultados se menciona la importancia de las organizaciones para impulsar la utilización del conocimiento académico por parte de los docentes, asumiendo que estas ofrecen un apoyo débil en esta acción. A propósito de las barreras que enfrenta el profesorado universitario a la hora de utilizar la investigación educativa, es importante reconocer que en el contexto nacional no siempre están condiciones que permitan compatibilizar la docencia con la investigación. La precarización contractual o la gran cantidad de horas de clases dificultan la apropiación y utilización de la investigación educativa por parte de los académicos.

Por otra parte, y desde la perspectiva de la formación inicial de profesores, es interesante mencionar el caso de Europa, donde la necesidad de promover la reflexión y la investigación en los currículos de Formación Inicial de Profesores (Initial Teacher Education) sigue siendo un problema sin resolver (Conway et al., 2009; Murray y Passy, 2014). En este sentido, es interesante la aportación de Flores et al. (2016) respecto al papel de la investigación en la formación práctica de los maestros con posterioridad al plan Bolonia implementado en Europa. En el estudio, los futuros profesores reconocen el valor educativo de los proyectos de investigación. Sin embargo, el surgimiento de una cultura orientada a la utilización de la investigación en la práctica necesita de mejoras y de una mayor reflexión para concretarla. La realización de actividades propias de la investigación educativa permite a los futuros profesores adquirir, y actualizar sus conocimientos (Cochran-Smith y Lytle, 2009). Si los profesores realizan investigaciones y utilizan sus resultados, tienen posibilidades de realizar mejoras para en propia práctica una vez que ingresen al mundo laboral (Gore y Gitlin, 2004).

La importancia de la formación en investigación educativa para desarrollar pensamiento crítico es otro de los elementos destacados en la literatura. De acuerdo con Huber y Kuncel (2016) el pensamiento crítico es una habilidad esencial en la trayectoria formativa de un estudiante de pregrado. Se reconoce cada vez más que la introducción de la literatura de investigación a los estudiantes universitarios les ayuda a convertirse en pensadores críticos (Carson, 2015; Sato et al., 2014). En esta misma línea, Wei et al. (2019) argumentan que una de las mejores maneras de preparar a los estudiantes para los cambios en las prácticas educativas es ayudarles a comprender de dónde provienen las mejores prácticas, lo que implica la revisión de la investigación existente en educación.

\section{METODOLOGÍA}

La presente investigación tiene como objetivo general analizar las valoraciones que los profesores universitarios de las pedagogías tienen sobre la formación en investigación educativa de los futuros docentes. Por su parte, los objetivos específicos del estudio son: 1) Determinar las valoraciones de los docentes universitarios sobre la importancia de la formación en investigación educativa de los futuros profesores; 2) Determinar las valoraciones de los profesores universitarios sobre la calidad de la formación en investigación educativa que se recibe actualmente en las pedagogías; 3) Determinar las valoraciones de los docentes universitarios sobre su disposición para proporcionar una formación en investigación educativa a los futuros profesores; 4) Determinar las valoraciones de los docentes universitarios sobre la disposición de la Universidad para proporcionar la formación en investigación educativa de los futuros profesores. El enfoque metodológico utilizado en este estudio es mixto. La utilidad de este enfoque radica en la utilización de las técnicas de investigación cuantitativa y cualitativa en un solo estudio de forma complementaria (Johnson y Onwuegbuzie, 2004; Pereira, 2011). En el caso del enfoque cuantitativo se llevó a cabo un estudio descriptivo ex post facto mediante el uso de un cuestionario. En el caso del enfoque cualitativo se realizaron 24 entrevistas semi-estructuradas.

\section{Participantes}

En el caso del estudio ex post facto, la muestra surge a partir de la población de docentes universitarios de la Universidad de La Serena, Chile, (también llamados académicos) que dan clases en alguna de las once carreras de pedagogía. La población es de 191 académicos de los cuales 77 respondieron el cuestionario $(40,3 \%)$. La aplicación se hizo de forma online a través de la plataforma informática de la Universidad. Se les solicitó su participación voluntaria vía correo electrónico institucional junto al respectivo consentimiento informado. En el caso del enfoque cualitativo, la selección de los participantes de las entrevistas semiestructuradas se realizó a través de un muestreo no probabilístico por cuotas. Los 24 académicos seleccionados se dividen de acuerdo con el nivel de experiencia que tiene como investigador: académicos que no han publicado artículos (8); académicos que se están iniciando en la publicación de artículos, un artículo en el último año (8), y académicos que tienen experiencia en publicaciones, dos o más artículos en el último año (8). 


\section{Instrumentos}

En el estudio ex post facto se construyó el instrumento denominado "cuestionario de valoraciones docentes" (que se adjunta en los anexos de este artículo), que es una adaptación de dos instrumentos de Griffioen (2018) y Borg (2009) aplicados anteriormente en otros contextos. Las variables a considerar en el cuestionario incluyen una primera parte de preguntas generales referidas a: 1) Años de experiencia en la docencia universitaria (de 0 a 5 años, de 6 a 15, más de 20 años de experiencia); 2) La asignatura donde da clases (área disciplinar-didáctica, área general o pedagógica, área de formación práctica); 3) Su relación con la publicación de investigación (no ha publicado, es investigador novel, es investigador experimentado).

La segunda parte del instrumento considera cuatro variables en coherencia a los cuatro objetivos específicos: 1) Valoraciones sobre la importancia de la formación en investigación educativa (siete enunciados); 2) Valoraciones sobre la calidad de la formación en investigación educativa que reciben actualmente los estudiantes de pedagogía (cinco enunciados); 3) Valoraciones sobre la disposición de los académicos para proporcionar una formación en investigación educativa (seis enunciados); 4) Valoraciones sobre la disposición de la Universidad para favorecer la formación en investigación educativa (seis enunciados). Estas cuatro variables son desglosadas por medio de afirmaciones con opción de respuesta en escala Likert de 1 a 5 . La validación del instrumento se realizó por medio del juicio de expertos (a priori) y de una validación experimental (a posteriori) que se detallará en el apartado de análisis de datos. Para la validación por juicio de expertos se contactó a diez investigadores, quienes realizaron críticas a los enunciados y a la estructura general del cuestionario. Una vez recogidas las aportaciones de los expertos se optimizó el instrumento para ser enviado a los académicos.

En el caso del enfoque cualitativo se utilizó la entrevista semi-estructurada. En coherencia con los objetivos específicos, algunas de las preguntas formuladas a los participantes son: 1) ¿Crees que es importante la formación en investigación educativa de los futuros docentes?; 2) ¿Cómo crees que es esta formación actualmente?; 3) ¿Crees que los formadores de profesores están preparados para proporcionar una formación en investigación educativa?; 4) ¿Qué gestiones puede realizar la institución para mejorar la formación en investigación educativa de los futuros docentes?

\section{Análisis de los datos}

El análisis cuantitativo de los datos se apoyó en el programa SPSS v.26. Las operaciones que se utilizaron fueron, entre otras, el cálculo de la fiabilidad, consistencia interna, análisis descriptivos (frecuencias) y análisis inferenciales (Anova de un factor). De los 77 académicos que respondieron el cuestionario, el 18,2 \% tiene menos de 5 años de experiencia en docencia universitaria, el 37,7\% tiene entre 6 y 15 años y el $44,2 \%$ cuenta con más de 15 años de experiencia. Respecto al área en la cual dan clases, el 58,4\% pertenece al área disciplinar-didáctico, el $22,1 \%$ al área de formación general o pedagógica y el 19,5\% pertenece al área de formación práctica. En cuanto a su relación con la publicación de investigaciones, el $46 \%$ de ellos afirma que aún no ha publicado artículos de investigación, el 15\% señala que en el último año publicó un artículo de investigación y el $16 \%$ publicó dos o más artículos de investigación en el mismo periodo.

\section{Consistencia interna del cuestionario}

El alfa de Cronbach obtenido para el cuestionario con sus 24 afirmaciones es de $\alpha=0,91$. La fiabilidad de cada subescala es: a) importancia de la formación en investigación educativa $\alpha=0,89$; b) calidad de la formación en investigación educativa $\alpha=0,95 ; c)$ Disposición de los académicos para proporcionar una formación en investigación educativa $\alpha=0,85$; y d) Disposición de la Universidad para favorecer la formación en investigación educativa $\alpha=0,87$. Considerando que el alfa de Cronbach de la escala obtiene una buena puntuación se procedió a realizar el análisis factorial. La tabla de comunalidades extraída del análisis factorial confirma que los 24 factores tienen una extracción que fluctúa entre 0,51 y 0,89 por lo que no se elimina ninguno de los ítems. Asimismo, la matriz de componentes confirma la existencia de cuatro subescalas. Los porcentajes de varianza explicados aportan información de la cantidad de varianza total explicada por cada factor, así como el porcentaje de varianza explicada total y acumulada. En este caso, se extraen cuatro factores que explican el $69,90 \%$ de la varianza total (tabla 1 ).

Por otra parte, el análisis cualitativo de los datos fue abordado desde un enfoque que recupera muchos de los postulados de la Teoría Fundamentada (Glaser y Strauss, 1967), la que sugiere un análisis de la información por medio de segmentos del discurso con significado propio. Lo que este estudio toma específicamente de la Teoría Fundamentada es la codificación y la comparación constante entre los códigos que se formulan. Los fragmentos de información se separan del resto del texto para ser analizados y codificados. Posteriormente, los códigos se ordenan y jerarquizan para facilitar la redacción de los resultados. En todo este proceso nos apoyamos en el programa Atlas.ti v.8, software que facilitó el análisis y la organización visual de la información. 
Tabla 1: Varianza explicada por cada factor.

\begin{tabular}{|l|c|c|c|}
\hline & Total T & $\%$ varianza & $\%$ acumulado \\
\hline $\begin{array}{l}\text { 1. Valoraciones sobre la importancia de la formación en } \\
\text { investigación educativa }\end{array}$ & 5,267 & 21,945 & 21,945 \\
\hline $\begin{array}{l}\text { 2. Valoraciones sobre la calidad de la formación en } \\
\text { investigación educativa }\end{array}$ & 4,608 & 19,201 & 41,146 \\
\hline $\begin{array}{l}\text { 3. Valoraciones sobre la disposición de los académicos para } \\
\text { proporcionar una formación en investigación educativa }\end{array}$ & 4,309 & 17,954 & 59,100 \\
\hline $\begin{array}{l}\text { 4. Valoraciones sobre la disposición de la Universidad para } \\
\text { proporcionar una formación en investigación educativa }\end{array}$ & 2,593 & 10,805 & 69,905 \\
\hline
\end{tabular}

\section{RESULTADOS}

Para exponer los resultados primero se muestran los hallazgos del cuestionario de valoraciones docentes y luego los resultados de las entrevistas. Del objetivo general "analizar las valoraciones que los profesores universitarios de las pedagogías tienen sobre la formación en investigación educativa de los futuros docentes" se desprenden los objetivos específicos 1, 2, 3 y 4, correspondientes a cada una de las subescalas del cuestionario. La media de las 24 afirmaciones de la escala Likert es de 3,56 puntos, lo que refleja, en general, una buena valoración hacia la formación en investigación educativa de los futuros docentes.

La afirmación que demuestra la media más alta $(m=4,78)$ es: "la investigación educativa es fundamental para que los/las futuros/as profesores/as se mantengan actualizados sobre los avances en materias de educación". Por el contrario, la afirmación que presenta la media más baja $(m=2,55)$ es: "en la Universidad donde trabajo hay una cultura investigadora que favorece la formación en investigación educativa de los futuros profesores". Respecto a cada subescala, se realizó una agrupación visual de los ítems para facilitar su interpretación, lo que se muestra en las Figuras 1, 2, 3 y 4.

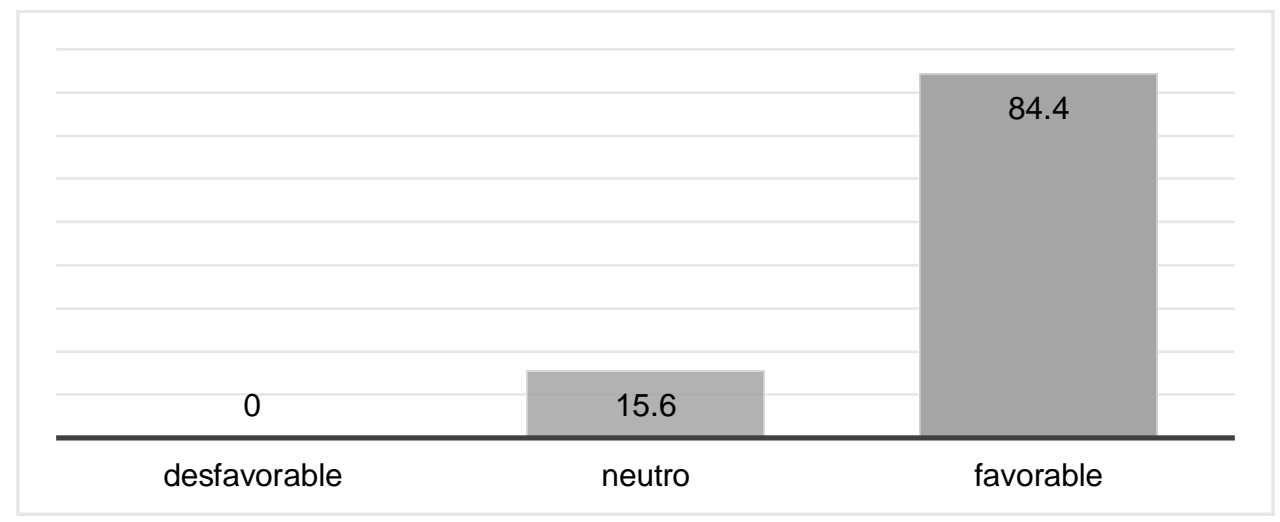

Fig 1: Valoraciones sobre la importancia de la formación en investigación educativa de los futuros profesores.

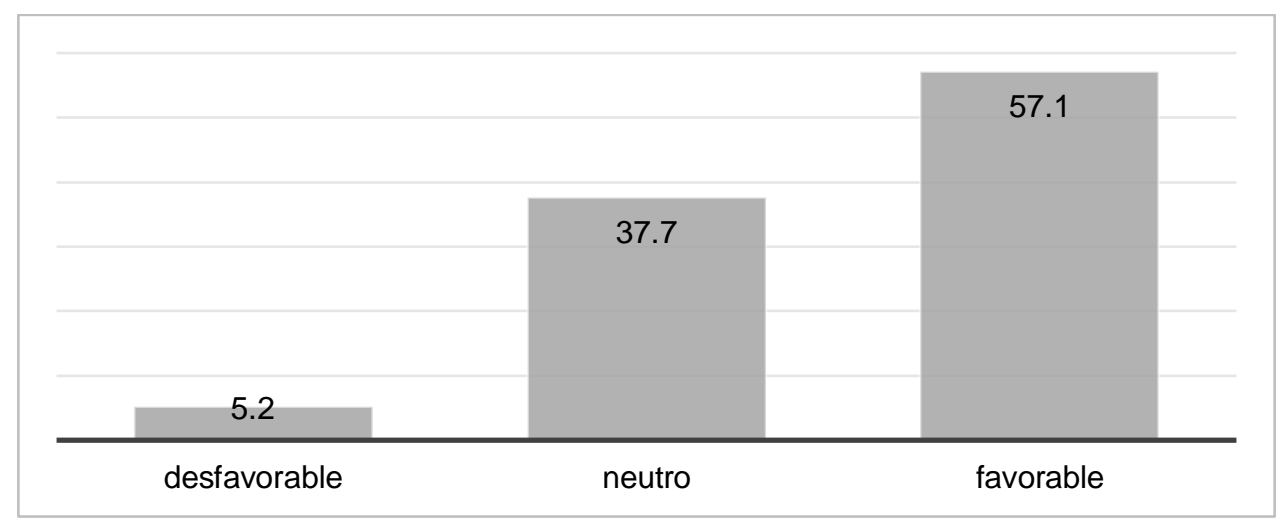

Fig 2: Valoraciones sobre la calidad de la formación en investigación educativa. 


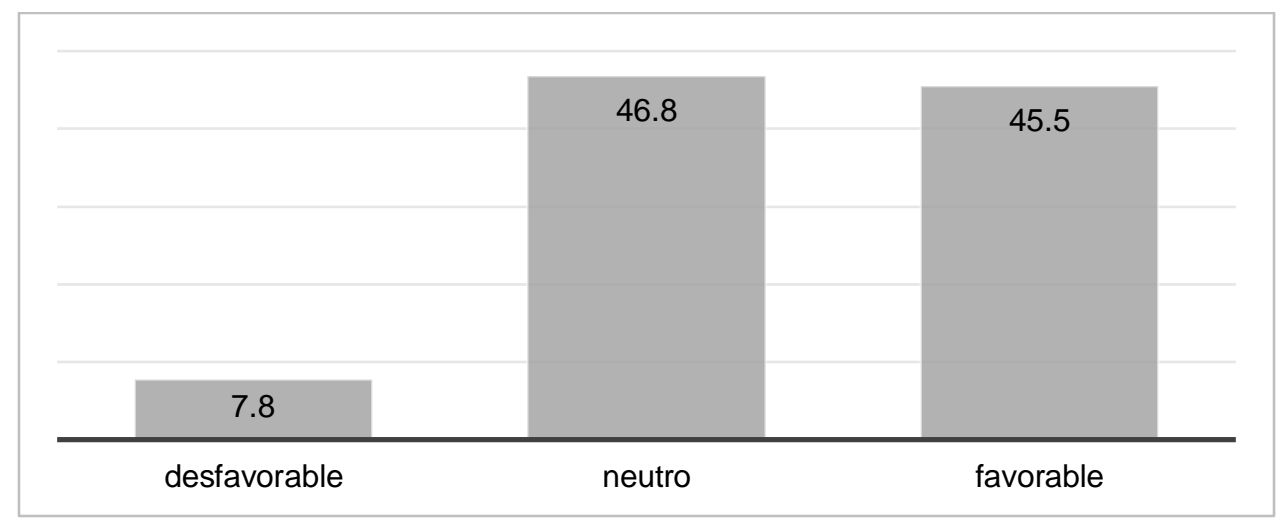

Fig 3: Valoraciones sobre la disposición de los académicos para proporcionar una formación en investigación educativa.

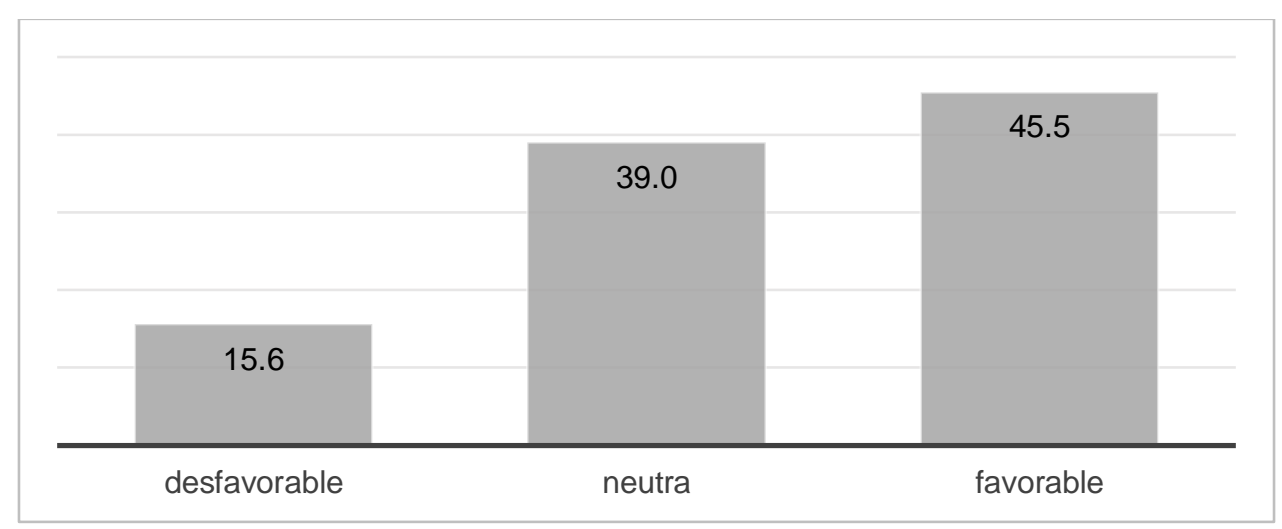

Fig 4: Valoraciones sobre la disposición de la Universidad para proporcionar una formación en investigación educativa.

En las figuras expuestas, queda en evidencia que la subescala 1 "importancia de la formación en investigación educativa" es la que obtiene mejores valoraciones, mientras que la que tiene opiniones más desfavorables es la subescala 4 "disposición de la universidad para proporcionar una formación en investigación educativa". Asimismo, se intentó determinar si existen diferencias en las cuatro subescalas en función de los años de experiencia en docencia universitaria de los académicos, el área en la cual dan clases y la experiencia en publicación de artículos de investigación, para lo cual se utilizó la función ANOVA de un factor y las comparaciones múltiples de Tukey que se muestran en las Tablas 2 y 3.

Tabla 2: Anova de un factor para las cuatro subescalas.

\begin{tabular}{|c|c|c|c|c|c|c|}
\hline \multicolumn{7}{|l|}{ ANOVA } \\
\hline & & $\begin{array}{l}\text { Suma de } \\
\text { cuadrados }\end{array}$ & gl & \begin{tabular}{|l|} 
Media \\
cuadrática
\end{tabular} & $\mathrm{F}$ & Sig. \\
\hline \multirow{3}{*}{$\begin{array}{l}\text { Subescala 1, importancia de la formación } \\
\text { en investigación educativa }\end{array}$} & Entre grupos & 2,037 & 2 & 1,018 & 4,158 & ,019 \\
\hline & Dentro de grupos & 18,122 & 74 & ,245 & & \\
\hline & Total & 20,159 & 76 & & & \\
\hline \multirow{3}{*}{$\begin{array}{l}\text { Subescala } 2 \text {, calidad de la formación en } \\
\text { investigación educativa }\end{array}$} & Entre grupos & 8,027 & 2 & 4,014 & 4,387 & ,016 \\
\hline & Dentro de grupos & 67,702 & 74 & ,915 & & \\
\hline & Total & 75,730 & 76 & & & \\
\hline \multirow[t]{3}{*}{ Subescala_3_disposición_académicos } & Entre grupos & 1,402 & 2 & ,701 & 1,138 & ,326 \\
\hline & Dentro de grupos & 45,602 & 74 &, 616 & & \\
\hline & Total & 47,004 & 76 & & & \\
\hline \multirow[t]{3}{*}{ Subescala_4_disposición_universidad } & Entre grupos &, 587 & 2 & ,293 & ,437 & ,648 \\
\hline & Dentro de grupos & 49,670 & 74 & ,671 & & \\
\hline & Total & 50,257 & 76 & & & \\
\hline
\end{tabular}


Tabla 3: Comparaciones múltiples de Tukey entre las 4 subescalas

\begin{tabular}{|c|c|c|c|c|c|c|c|}
\hline \multicolumn{8}{|l|}{ Comparaciones múltiples } \\
\hline \multirow[t]{2}{*}{ Variable dependiente } & \multirow[t]{2}{*}{$\begin{array}{l}\text { (I) años de } \\
\text { experiencia }\end{array}$} & \multirow[t]{2}{*}{$\begin{array}{l}\text { (J) años de } \\
\text { experiencia }\end{array}$} & \multirow[t]{2}{*}{$\begin{array}{l}\text { Diferencia de } \\
\text { medias (I-J) }\end{array}$} & \multirow[t]{2}{*}{$\begin{array}{l}\text { Error } \\
\text { estándar }\end{array}$} & \multirow[t]{2}{*}{ Sig. } & \multicolumn{2}{|c|}{$\begin{array}{l}\text { Intervalo de } \\
\text { confianza al 95\% }\end{array}$} \\
\hline & & & & & & $\begin{array}{l}\text { Límite } \\
\text { inferior }\end{array}$ & $\begin{array}{l}\text { Límite } \\
\text { superior }\end{array}$ \\
\hline \multirow{6}{*}{$\begin{array}{l}\text { Subescala } 1 \text {, importancia } \\
\text { de la formación en } \\
\text { investigación educativa }\end{array}$} & \multirow[t]{2}{*}{ De 0 a 5 años } & De 6 a 15 años &, $407^{*}$ & ,161 & ,036 & ,02 & ,79 \\
\hline & & $\begin{array}{l}\text { Más de } 20 \text { años } \\
\text { de experiencia }\end{array}$ &, $432^{\star}$ &, 157 &, 020 &, 06 & ,81 \\
\hline & \multirow[t]{2}{*}{ De 6 a 15 años } & De 0 a 5 años &,$- 407^{\star}$ &, 161 & ,036 &,- 79 &,- 02 \\
\hline & & $\begin{array}{l}\text { Más de } 20 \text { años } \\
\text { de experiencia }\end{array}$ &, 025 & ,125 & ,978 &,- 27 & ,32 \\
\hline & \multirow{2}{*}{$\begin{array}{l}\text { Más de } 20 \text { años } \\
\text { de experiencia }\end{array}$} & De 0 a 5 años &,$- 432^{*}$ & ,157 &, 020 &,- 81 &,- 06 \\
\hline & & De 6 a 15 años &,- 025 & ,125 & ,978 &,- 32 & ,27 \\
\hline \multirow{6}{*}{$\begin{array}{l}\text { Subescala } 2 \text {, calidad de la } \\
\text { formación en investigación } \\
\text { educativa }\end{array}$} & \multirow[t]{2}{*}{ De 0 a 5 años } & De 6 a 15 años &, $92167^{\star}$ & ,31128 &, 011 &, 1772 & 1,6662 \\
\hline & & $\begin{array}{l}\text { Más de } 20 \text { años } \\
\text { de experiencia }\end{array}$ &, 64034 & ,30374 &, 095 &,- 0861 & 1,3668 \\
\hline & \multirow[t]{2}{*}{ De 6 a 15 años } & De 0 a 5 años &,$- 92167^{\star}$ & ,31128 &, 011 & $-1,6662$ &,- 1772 \\
\hline & & $\begin{array}{l}\text { Más de } 20 \text { años } \\
\text { de experiencia }\end{array}$ &,- 28134 & ,24178 & ,479 &,- 8596 & ,2969 \\
\hline & \multirow{2}{*}{$\begin{array}{l}\text { Más de } 20 \text { años } \\
\text { de experiencia }\end{array}$} & De 0 a 5 años &,- 64034 & ,30374 &, 095 & $-1,3668$ & 0861 \\
\hline & & De 6 a 15 años & ,28134 & ,24178 & ,479 &,- 2969 & ,8596 \\
\hline
\end{tabular}

Tal como se puede observar en las Tablas 2 y 3 , en el caso de la experiencia en docencia universitaria, se encontraron diferencias significativas en las valoraciones sobre la importancia de la formación en investigación educativa y sobre la calidad de dicha formación. Específicamente, los académicos con menos experiencia en docencia universitaria (entre 0 y 5 años) tuvieron mejores valoraciones sobre la importancia de la formación en investigación educativa como se puede observar en la Tabla $4(\mathrm{M}=4,95)$. Lo mismo sucede con la calidad de la formación en investigación educativa, donde nuevamente los académicos con menos años de experiencia manifiestan mejores valoraciones como se puede observar en la Tabla $5(M=4,12)$.

Tabla 4: Diferencias en la subescala 1 respecto a los años de experiencia en docencia en educación superior.

\begin{tabular}{|l|l|l|l|}
\hline \multicolumn{4}{|l|}{ Subescala 1, importancia de la formación en investigación educativa } \\
\hline HSD Tukeya,b & N & \multicolumn{2}{|l|}{ Subconjunto para alfa $=0.05$} \\
\cline { 3 - 5 } & & 1 & 2 \\
\hline Años de experiencia & 34 & 4,52 & \\
\hline Más de 20 años de experiencia & 39 & 4,54 & 4,95 \\
\hline De 6 a 15 años & 29 & 1,000 \\
\hline De 0 a 5 años & 14 & &, 984 \\
\hline Sig. & & & \\
\hline
\end{tabular}

No se encontraron diferencias significativas respecto al área en el cual los académicos dan clases ni tampoco en cuanto de acuerdo con la experiencia en publicación de artículos de investigación. Por otra parte, las entrevistas realizadas a los profesores universitarios identificaron cuatro temas centrales: los beneficios de la formación en investigación educativa, la situación actual de la formación en investigación educativa de los futuros profesores, el rol de las instituciones para lograr una formación en investigación educativa y el rol de los propios profesores universitarios (que los participantes denominan "académicos" como ya se mencionó). Estos cuatro temas derivan de las familias semánticas identificadas en el análisis de los discursos y en las cuales hay una serie de códigos asociales, lo que se muestra en la Figura 5. 
Tabla 5: Diferencias en la subescala 2 respecto a los años de experiencia en docencia en educación superior.

\begin{tabular}{|l|l|c|c|}
\hline \multicolumn{3}{|l|}{ Subescala 2, calidad de la formación en investigación educativa } \\
\hline HSD Tukeya,b & $\mathrm{N}$ & \multicolumn{3}{|c|}{ Subconjunto para alfa $=0.05$} \\
\cline { 3 - 4 } & & 1 & 2 \\
\hline años de experiencia & 29 & 3,2069 & \\
\hline De 6 a 15 años & 34 & 3,4882 & 3,4882 \\
\hline Más de 20 años de experiencia & 14 & & 4,1286 \\
\hline De 0 a 5 años & &, 592 &, 073 \\
\hline Sig.
\end{tabular}
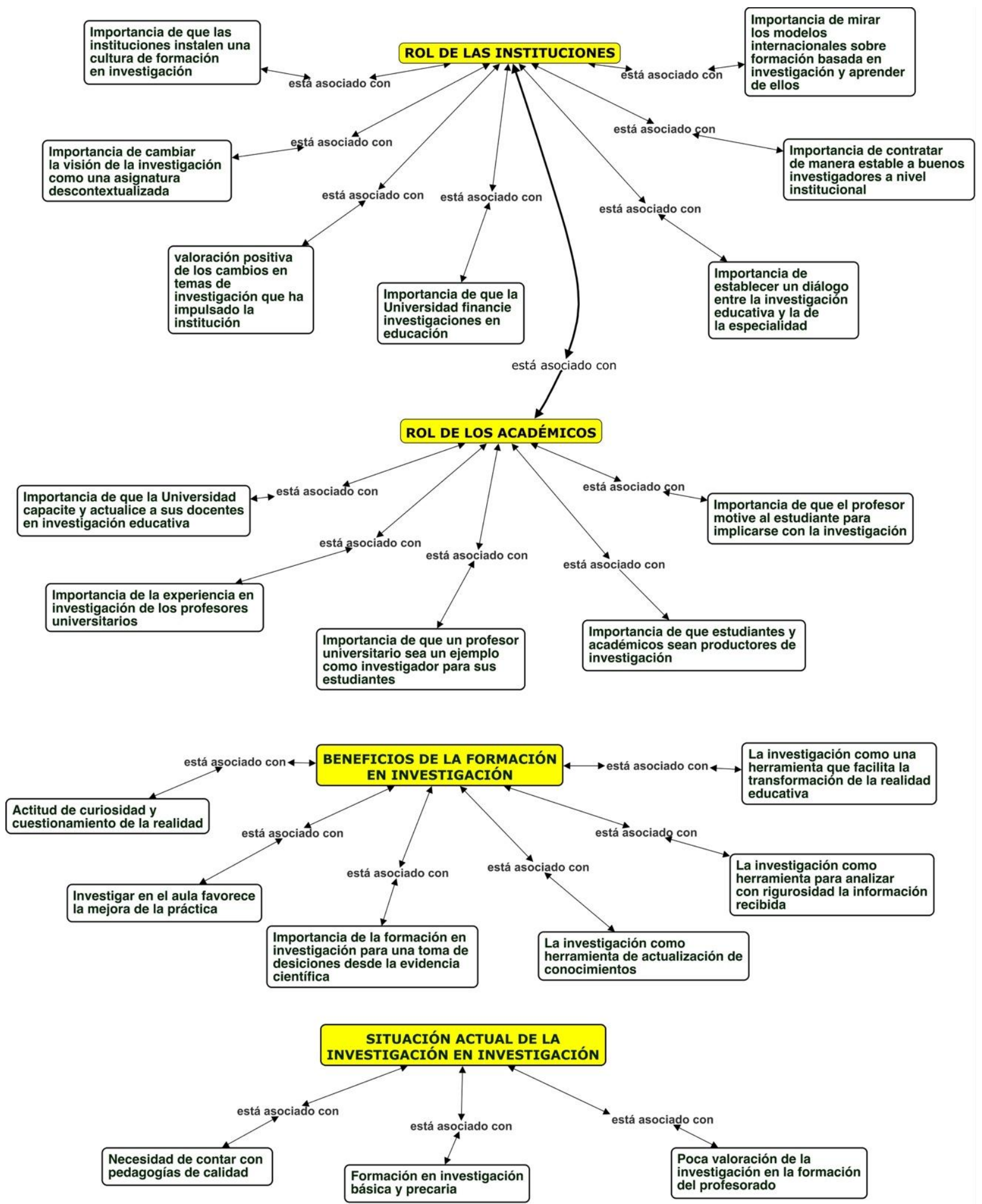

Fig 5: Familias semánticas y códigos derivados de las entrevistas a profesores universitarios (software Atlas.ti.8. 


\section{Beneficios de la formación en investigación educativa}

Los participantes, sin diferencias relevantes de acuerdo con su experiencia como investigadores, coindicen en que la formación en investigación educativa de los futuros profesores es muy importante e incluso fundamental para su quehacer pedagógico. Mencionan una serie de beneficios que tendría la formación en investigación educativa para un estudiante de pedagogía, por ejemplo, que a través de ella se actualizan conocimientos tanto en la propia formación como en la futura práctica laboral y que les permite desarrollar una actitud crítica de curiosidad y cuestionamiento de la realidad educativa: Valorar la investigación como una forma de contribuir a la mejora continua de su práctica que tiene que ver con mantenerse al día en lo más avanzado de la disciplina y eso va contribuyendo a no quedarse en lo tradicional, a mantenerse actualizado.

A propósito de la importancia de una buena toma de decisiones, para algunos de los participantes es importante tomar en cuenta las evidencias que proporciona la investigación educativa, lo que favorece una mirada más rigurosa frente a la información recibida. Algunos de ellos critican que el profesorado oriente sus acciones a través del sentido común y no por medio de datos provenientes de la investigación. También se trata de no "comprar" cualquier información de cualquier parte, sino que contar con herramientas para filtrar ¿qué cosa me están llegando?, ¿eso tiene una validez?, y no leer cualquier cosa por ahí y creer que es verdad y después aplicarla.

\section{Situación actual de la formación en investigación educativa.}

El segundo tema identificado en el discurso de los académicos/as es la descripción más bien crítica que estos realizan sobre la situación actual de la formación en investigación de los futuros profesores. Por ejemplo, algunos de ellos comparan a la medicina con la pedagogía, destacando que ambas áreas tienen una forma abismalmente diferente de hacer uso de la investigación. Las palabras de uno de los participantes sirven para ejemplificarlo: es lo que hace el médico... muchos médicos tal vez no investigan, pero sí hacen uso de la investigación que está disponible para mejorar su práctica profesional y atender mejor a sus pacientes (...) pero eso no pasa con los profesores. Los médicos te recomiendan un tratamiento y van evaluando si este tratamiento funcionó en ti y si te ayudó a mejorar tu salud. Lo mismo debería pasar en la pedagogía, pero no sucede. Asimismo, algunos participantes afirman que, en el contexto universitario actual, la formación en investigación educativa es básica y precaria. La principal crítica es que no existe una vinculación entre las asignaturas de investigación en los programas. Otras críticas apuntan a que la formación en investigación educativa actual es teórica y no permite que los profesores cuenten con herramientas para mejorar la calidad de la enseñanza cuando se conviertan en docentes en ejercicio. Los programas actuales en temas de formación de profesores ofrecen una que otra asignatura donde se puede aprender algo sobre la investigación. Pero en general son demasiado teóricas. Esto no permite que los estudiantes conozcan a la investigación de manera tangible.

\section{Rol de las instituciones en la formación en investigación educativa}

Gran parte de las opiniones mencionan la importancia de que las universidades realicen acciones concretas, como, por ejemplo, contratar de manera estable a buenos docentes-investigadores y por sobre todo capacitar y actualizar a los académicos en temas de investigación educativa: Primero creo que la universidad podría dar capacitaciones para empezar a hacer investigación en educación. Creo que es necesario que nos capaciten, porque va a ver mucha gente que sí va a querer hacerlo. Por otro lado, hay académicos que consideran importante que al interior de las pedagogías se establezca un mayor diálogo entre el área de la especialidad (matemáticas, lenguaje, biología) y el área de la pedagogía. Para algunos de ellos mejorar la forma en que se comunican estas áreas favorecería que la formación en investigación educativa de los futuros profesores fuera más transversal e intencionada al interior de las carreras.

Asimismo, surge la opinión generalizada de instalar una cultura investigativa en las universidades, lo que implica modificar los modelos ya existentes en temas de formación de profesorado e instalar políticas de investigación estables y permanentes. A propósito de ello algunos participantes mencionan la importancia de observar modelos internacionales sobre formación basada en investigación y aprender de ellos como, por ejemplo, la instauración semilleros de investigación. La carrera de Educación Parvularia de la Universidad donde se realiza este estudio cuenta con un semillero lo que es valorado positivamente por académicos de otras carreras como un ejemplo a seguir: Por ejemplo, en esta Universidad hay una carrera que cuenta con un semillero de investigación desde hace muchos años. Ojalá todas las carreras de pedagogía hicieran lo mismo. Es una excelente vía para formar a los estudiantes en investigación.

En el discurso de los académicos también surgen valoraciones positivas hacia los cambios que la institución donde trabajan está impulsando en materias de formación en investigación educativa. Por ejemplo, se valora la renovación curricular de las once carreras de pedagogía implementada desde el año 2019 donde se da mayor énfasis a la formación investigativa de los futuros profesores. Destacan que la renovación potencie las 
asignaturas vinculadas a la investigación, que se les dé una gradualidad y que se fortalezca la licenciatura en educación. También se valora positivamente que la universidad ofrezca concursos internos donde se entregue financiamiento para ejecutar una investigación. La universidad está haciendo cambios importantes, por ejemplo, se renovaron las once pedagogías y en estos cambios se vislumbra una mayor inclusión de la investigación, hay más asignaturas y están más conectadas. También se ofrecen concursos de investigación a los cuales se puede postular y conseguir financiamiento. Son cosas que se valoran y que se deberían ser más aprovechadas por los académicos.

\section{El rol de los propios académicos}

En las entrevistas realizadas, los académicos mencionan que formar a los estudiantes de pedagogía en investigación requiere que los académicos tengan experiencia en proyectos y publicaciones: Hay muchos recursos humanos que no están capacitados simplemente para poder enseñar a investigar, porque ellos mismos no tienen experiencia en investigación. Incluso, para algunos de los participantes la realización de postgrados también puede ser un factor determinante para la forma en que los académicos abordan la formación en investigación educativa. Hay profesores universitarios que sí tienen un doctorado, que efectivamente hacen investigación, y claro, para ellos es importante transmitir también los parámetros de investigación, exigen a sus estudiantes por ejemplo que tengan una escritura más académica, que usen norma APA, que busquen en base de datos. Otros académicos mencionan que los profesores universitarios deben ser un ejemplo para los estudiantes en materias de investigación y deben ser consecuentes entre lo que dicen y lo que hacen, lo que incluye la actividad investigadora. Si nosotros los profesores realmente creemos que la investigación es muy importante podemos actuar como modelo para los alumnos, por ejemplo, para un alumno leer un paper que hizo un profesor, y decir: el "profe" lo escribió y lo publicó.

\section{DISCUSIÓN}

Un primer elemento para discutir en el presente artículo es la forma en que dialogan los hallazgos del estudio ex post facto y el estudio cualitativo. Lo que queda claro es que, en ambos casos, se considera fundamental que los estudiantes de pedagogía reciban formación en investigación educativa. En cuanto a la calidad de dicha formación las coincidencias son menos precisas. Los académicos de los cuestionarios manifiestan en general una buena opinión de dicha calidad, mientras que los participantes de las entrevistas valoran los avances, pero asumen que todavía queda mucho por hacer. Respecto a la disposición de los académicos y de la universidad para proporcionar una formación en investigación educativa, existen coincidencias ya que, en general, las valoraciones de los académicos son más críticas en estos dos aspectos, aunque no de una manera radical.

Al analizar estos resultados respecto a la literatura vigente, vemos que, en coherencia a lo planteado por Larenas et al. (2015) y Rubio et al. (2018), los académicos consideran que formar a los futuros profesores en temas de investigación educativa es muy necesario e importante. Sin embargo, asumen ciertas dificultades para lograrlo. Por ejemplo, algunos de los participantes critican que los docentes no recurran a la evidencia para fundamentar sus decisiones (a diferencia de los médicos), con lo cual la comparación entre la medicina y la enseñanza realizada por Hargreaves (1996) cobra sentido. Asimismo, algunos de los académicos de este estudio mencionan que los profesores universitarios deben hacer el esfuerzo por también ser investigadores o al menos consumidores de investigación, lo que va en coherencia al necesario vínculo docenciainvestigación que menciona Tesouro et al. (2014). Esta contrastación con la teoría permite ratificar la importancia de la investigación educativa en la formación de profesorado y asumir los desafíos que todavía quedan pendientes para instalarla adecuadamente en los programas de pedagogía. En este sentido, cobra relevancia lo señalado por Flores et al. (2016), cuando reconocen que el surgimiento de una cultura requiere de mayores esfuerzos y reflexiones.

Las principales limitaciones del estudio son haber contado con una muestra pequeña y haber aplicado el cuestionario solo en una Universidad. Superar estas limitaciones permite proyectar este estudio en un conjunto de universidades, incluso, se puede comparar lo que sucede en universidades públicas y privadas. También se puede optimizar el cuestionario de que se pone a disposición de la comunidad científica como un aporte a la literatura sobre el tema y que puede ser utilizado por investigadores de otras latitudes.

\section{CONCLUSIONES}

De acuerdo con los resultados, su análisis, discusión y en coherencia a los objetivos específicos se pueden obtener las siguientes conclusiones:

1. Respecto al objetivo específico 1 (Determinar las valoraciones de los docentes universitarios sobre la importancia de la formación en investigación educativa de los futuros profesores) se concluye que la formación es investigación educativa de los futuros profesores es muy importante para los formadores de profesores. 
Así lo refleja que la afirmación con la media más alta fuera "la investigación educativa es fundamental para que los/las futuros/as profesores/as se mantengan actualizados sobre los avances en materias de educación".

2. En relación con objetivo específico 2 (Determinar las valoraciones de los profesores universitarios sobre la calidad de la formación en investigación educativa que se recibe actualmente en las pedagogías) es posible concluir que la calidad de la formación en investigación educativa se debe optimizar. Los esfuerzos para que los futuros profesores tengan una mayor vinculación con la investigación educativa no pueden quedarse solo en un plano instrumental. Si el esfuerzo por parte de los académicos y de las instituciones apunta a que un estudiante se convierta en un experto en metodología de la investigación descontextualizada de la realidad de las escuelas nos quedamos solo en un plano abstracto de la investigación que desconoce las culturas escolares.

3. Respecto al objetivo específico 3 (Determinar las valoraciones de los docentes universitarios sobre su disposición para proporcionar una formación en investigación educativa a los futuros profesores) se concluye que los académicos deben optimizar su propia preparación en temas de investigación educativa. No solo a través de la obtención de grados académicos, sino que por medio de la concientización sobre la importancia que tiene la investigación educativa para la formación de un profesorado más crítico, informado y actualizado.

4. Finalmente, en lo que respecto al objetivo específico 4 (Determinar las valoraciones de los docentes universitarios sobre la disposición de la Universidad para proporcionar la formación en investigación educativa de los futuros profesores) las conclusiones son claras: el mayor desafío pendiente es optimizar la forma en que las Universidades disponen recursos y esfuerzos para favorecer una formación en investigación educativa. Recordemos que la afirmación "en la Universidad donde trabajo hay una cultura investigadora que favorece la formación en investigación educativa de los futuros profesores" es la que obtuvo peores valoraciones.

\section{AGRADECIMIENTOS}

La presente investigación surge desde el proyecto "Presencia e importancia de la investigación educativa en la formación pedagógica y su valoración por estudiantes y docentes" adjudicado por la autora y financiado por ANID, a través del proyecto Fondecyt de iniciación № 11180227.

\section{REFERENCIAS}

Admiraal, W., Buijs M. y otros 3 autores, Linking Theory and Practice: Teacher Research in History and Geography Classrooms, https://doi.org/10.1080/09650792.2016.1152904, Educational Action Research, 25(2), 316-331, (2017).

Aldana de Becerra, G. M., La Formación Investigativa: su Pertinencia en el Pregrado. Revista Virtual Universidad Católica del Norte, ISSN: 0124-5821 1(35), 367-379, (2012).

Anwaruddin, S. y Pervin, N., English-language Teachers' Engagement with Research: Findings from Bangladesh, https://doi.org/10.1080/19415257.2013.861860, Professional Development in Education, 41(1), 21-39, (2015).

Barkhuizen, G., Burns, A., Kenan, D. y Wyatt, M., Empowering Teacher Researchers Empowering Learners. Faversham: IATEFL. (2018).

Bartels, N., How Teachers and Researchers Read Academic Articles, https://doi.org/10.1016/j.tate.2003.06.001, Teaching and Teacher Education, 19(7), 737-753, (2003).

Borg, S., English Language Teachers' Conceptions of Research, https://doi.org/10.1093/applin/amp007, Applied Linguistics, 30(3), 358-388, (2009).

Broekkamp, H. y van Hout-Wolters, B., The gap Between Educational Research and Practice: A literature Review, Symposium, and Questionnaire, https://doi.org/10.1080/13803610701626127, Educational Research and Evaluation, 13(3), 203-220, (2007).

Burns, A., Dikilitaş, K., Smith, R. y Wyatt, M., Introduction. En A. Burns, K, Dikilitaş, R. Smith y M. Wyatt (Eds.), Developing Insights into Teacher-Research (pp. 1-17). Faversham: IATEFL. (2017).

Cardoso, E. O. y Cerecedo, M. T., Valoración de las Competencias Investigativas de los Estudiantes de Posgrado en Administración. https://doi.org/10.4067/s0718-50062019000100035, Formación Universitaria, 12(1), 35-44. (2019).

Carson, S., Targeting Critical Thinking Skills in a First-Year Undergraduate Research Course, https://doi.org/10.1128/jmbe.v16i2.935, Journal of Microbiology and Biology Education, 16(2), 148, (2015).

Conway, P., Murphy, R., Rath, A. y Hall, K., Learning to Teach and its Implications for the Continuum of Teacher Education: A Nine-Country Cross-National Study. Report Commissioned by the Teaching Council, University College, Cork, Ireland. (2009).

Cochran-Smith, M., y Lytle, S. L., Inquiry as Stance: Practitioner Research for the Next Generation. New York: Teachers College Press. (2009). 
Demircioglu, I. H., Learning How to Conduct Educational Research in Teacher Education: A Turkish Perspective, https://doi.org/10.14221/ajte.2008v33n1.1, Australian Journal of Teacher Education, 33(1), 1-17, (2008).

Díaz Costa, E., Cano, A. F., Nadim, T.F. y Carrillo, C.C., Modelamiento y Estimación del Índice de Impacto de la Investigación sobre la Docencia, https://doi.org/10.6018/reifop.22.2.351671, Revista Electrónica Interuniversitaria de Formación del Profesorado, 22(2), (2019).

Flores, M.A., Vieira, F., Silva, J.L. y Almeida, J., Integrating Research into the Practicum: Inquiring into Inquiry-Based Professional Development in post-Bologna Initial Teacher Education in Portugal. in M. A. Flores and T. Al-Barwani (Eds.), Redefining Teacher Education for the post-2015 Era: Global Challenges and Best Practice, (pp.109-124), New York: Nova Publisher. (2016)

Glaser, B., y Strauss, A., El Desarrollo de la Teoría Fundada. Chicago, EEUU: Aldine. (1967).

Gore, J.M. y Gitlin, A. D., [Re] Visioning the Academic-Teacher Divide: Power and Knowledge in the Educational Community. https://doi.org/10.1080/13540600320000170918, Teachers and Teaching, 10(1), 35-58, (2004).

Griffioen, D., Onderzoeksintegratie en de Intentie tot Onderzoeksgedrag van Studenten in Hoger Beroepsonderwijs (2018).

Haberfellner, C., y Fenzl, T., The Utility Value of Research Evidence for Educational Practice from the Perspective of Preservice Student Teachers in Austria-A Qualitative Exploratory Study. Journal for Educational Research Online/Journal für Bildungsforschung Online, 9(2), 69-87, (2017).

Hargreaves, D. H., Teaching as a Research-Based Profession: Possibilities and Prospects. The Teacher Training Agency, Anual Lecture. (1996).

Heggen, K., Karseth, B. y Kyvik, S., The Relevance of Research for the Improvement of Education and Professional Practice. The Research Mission of Higher Education Institutions Outside the University Sector. https://doi.org/10.1007/9781-4020-9244-2_3, (pp. 45-60). Springer, Dordrecht. (2010).

Hernández Arteaga, I., El Docente Investigador en la Formación de Profesionales. Revista Virtual Universidad Católica del Norte, ISSN: 0124-5821, 1(27), (2009).

Huber, C. R., y Kuncel, N. R., Does College Teach Critical Thinking? A Meta-Analysis, https://doi.org/10.3102/0034654315605917, Review of Educational Research, 86(2), 431-468, (2016).

Johnson, R. y Onwuegbuzie, A., Mixed Methods Research: A research Paradigm whose time has come, https://doi.org/10.3102/0013189X033007014, Educational Researcher, 33(7), 14-26, (2004).

Joram, E., Clashing Epistemologies: Aspiring Teachers', Practicing Teachers', and Professors' Beliefs about Knowledge and Research in Education, https://doi.org/10.1016/j.tate.2006.04.032, Teaching and Teacher Education, 23(2), 123-135, (2007).

Kaestle, C., The awful Reputation of Education Research, https://www.jstor.org/stable/1177303, Educational Researcher, 22(1), 23-31, (1993)

Larenas, C. H. D., Rodríguez, M. I. S. y otros tres autores, Temas Clave en la Formación de Profesores en Chile desde la Perspectiva de Docentes y Directivos, https://doi.org/10.5209/rev_rced.2015.v26.n3.44300, Revista Complutense de Educación, 26(3), 543, (2015).

Lazcano-Peña, D., Viedma G. C. y Alcaino, T. V., Comunicación de la Ciencia desde la Mirada de los Investigadores Universitarios: entre el Indicador y la Vocación. https://doi.org/10.4067/s0718-50062019000600027, Formación Universitaria, 12(6), 27-40, (2019).

Marsh, H. W., y Hattie, J., The Relation Between Research Productivity and Teaching Effectiveness, https://doi.org/10.1353/jhe.2002.0047, The Journal of Higher Education, 73(5), 603-641, (2002).

Murillo, F. J., y Perines, H., Cómo los Docentes no Universitarios Perciben la Investigación Educativa/How Non-University Teachers Perceive Educational Research, https://doi.org/10.5209/rev rced.2017.v28.n1.48800, Revista Complutense de Educación, 28(1), 81, (2017).

Murillo, F. J., Perines, H. y Lomba, L., La Comunicación de la Investigación Educativa. Una Aproximación a la Percepción de los Artículos Académicos y de Difusión por parte de Docentes no Universitarios. Profesorado. Revista de Curriculum y Formación de Profesorado, ISSN: 1989-6395, 21(3), 183-200, (2017).

Murray, J., y Passy, R., Primary Teacher Education in England: 40 years on, http://dx.doi.org/10.1080/02607476.2014.956542, Journal of Education for Teaching, 40(5), 492-506, (2014).

Pereira, Z., Los Diseños de Método Mixto en la Investigación en Educación: Una Experiencia Concreta. Revista Electrónica Educare, ISSN: 1409-42-58, 15(1), 15-29, (2011).

Perines, H., y Murillo, J., Percepciones de los Docentes en Formación sobre la Investigación Educativa, http://dx.doi.org/10.4067/S0718-07052017000100015, Estudios Pedagógicos (Valdivia), 43(1), 251-268 (2017).

Roche, L., y Marsh, H., Multiple Dimensions of University Teacher Self-Concept, https://doi.org/10.1023/A:1026576404113, Instructional Science, 28, 439-468, (2000).

Rubio, M.J., Torrado, M., Quirós, C. y Valls, R., Autopercepción de las Competencias Investigativas en Estudiantes de Último curso de Pedagogía de la Universidad de Barcelona para Desarrollar su Trabajo de Fin de Grado, https://doi.org/10.5209/RCED.52443, Revista Complutense de Educación, 29(2), 335, (2018). 
Sato, B. K., Kadandale P. y otros cuatro autores, Practice Makes Pretty Good: Assessment of Primary Literature Reading Abilities Across Multiple Large-Enrollment Biology Laboratory Courses, https://doi.org/10.1187/cbe.14-02-0025, CBELife Sciences Education, 13(4), 677-686, (2014).

Schouteden, W., Verburgh, A. y Elen, J., Teachers' General and contextualised Research Conceptions, https://doi.org/10.1080/03075079.2014.914915, Studies in Higher Education, 41(1), 79-94, (2016).

Smith, R., The International Festival of Teacher-Research in ELT: Richard Smith Interviewed by Deborah Bullock. ELT Research 33, 32-36, (2018).

Spronken-Smith, R., Walker, R. y otros tres autores, Evaluating Student Perceptions of Learning Processes and Intended Learning Outcomes Under Inquiry Approaches, https://doi.org/10.1080/02602938.2010.496531, Assessment and Evaluation in Higher Education, 37(1), 57-72, (2012).

Tesouro, M., Corominas, E., Teixidó, J. y Puiggalí, J., La Autoeficacia Docente e Investigadora del Profesorado Universitario: Relación con su Estilo Docente e Influencia en sus Concepciones sobre el Nexo Docencia Investigación, https://doi.org/10.6018/rie.32.1.172771, Revista de Investigación Educativa, 32 (1), 169-186, (2014).

van Schaik, P., Volman, M., Admiraal, W. y Schenke, W., Barriers and Conditions for Teachers' Utilisation of Academic Knowledge, https://doi.org/10.1016/j.ijer.2018.05.003, International Journal of Educational Research, 90, 50-63, (2018).

Wei, W., Hudson, M. A. y Cook A. E., Using Primary Research Literature to Teach Critical Thinking in Pre-Service Teacher Education, https://doi.org/10.4018/978-1-5225-7823-9.ch022, En Handbook of Research on Critical Thinking Strategies in Pre-Service Learning Environments (pp. 448-469). IGI Global (2019). 
